\title{
Chlorinated, Brominated and Perfluorinated Contaminants in Livers of Polar Bears from Alaska
}

\author{
KURUNTHACHALAM KANNAN ${ }^{*}$, , SE HUN YUN ${ }^{\dagger}$, THOMAS J. EVANS \\ †Wadsworth Center, New York State Department of Health and Department of Environmental \\ Health and Toxicology, School of Public Health, State University of New York at Albany, \\ Empire State Plaza, PO Box 509, Albany, New York 12201-0509, USA \\ ${ }^{\star}$ United States Fish and Wildlife Service, 1011 East Tudor Road, Anchorage, Alaska 99503, \\ USA
}

\section{Supplementary Information I.}

\section{Analysis of Organohalogens in Polar Bear Livers and QA/QC.}

Approximately $5 \mathrm{~g}$ of liver tissue was homogenized with anhydrous sodium sulfate and extracted in a Soxhlet apparatus for $16 \mathrm{~h}$, using dichloromethane and hexane $(3: 1 ; 400 \mathrm{~mL})$. The extract was rotary-evaporated at $40^{\circ} \mathrm{C}$ to $10 \mathrm{~mL}$, and a $1 \mathrm{~mL}$ aliquot of the extract was used for the determination of fat content by gravimetry. The remaining extract was spiked with ${ }^{13} \mathrm{C}$ labeled PCB congeners 3, 15, 31, 52, 118, 153, 180, 194, 206, 209, and ${ }^{13}$ C-labeled PBDE congeners $3,15,28,47,99,100,118$, and 153 as internal standards. PCB congeners $30(2,4,6-$ triCB) and $204\left(2,2^{\prime}, 3,4,4^{\prime}, 5,6,6^{\prime}\right.$-octaCB $)$ were spiked as surrogate standards. A portion of the sample extract was then purified by passage through a series of layers of silica gel (Davisil, 100200 mesh, Aldrich, WI; $1 \mathrm{~g}$ of silica gel, $2 \mathrm{~g}$ of $40 \%$ acidic-silica gel, $1 \mathrm{~g}$ of silica gel, $2 \mathrm{~g}$ of $40 \%$ acidic-silica gel, and $1 \mathrm{~g}$ of silica gel at the top). The extract was then treated with sulfuric acid $(5 \mathrm{~mL})$ and concentrated to $1 \mathrm{~mL}$ for the analysis of PCBs and PBDEs. Another portion of the extract was passed through silica gel ( $2 \mathrm{~g}$ ) by elution with $20 \%$ dichloromethane in hexane; it was then treated with sulfuric acid, for the analysis of organochlorine pesticides. 
Extracts were injected into a gas chromatograph (Hewlett-Packed 6890) coupled with a mass-selective detector (Hewlett-Packed, series 5973) for the determination of PCBs and PBDEs. A capillary column coated with RTX-5MS (30 $\mathrm{m}$ x $0.25 \mathrm{~mm}$ i.d. $\mathrm{x} 0.25 \mu \mathrm{m}$ film thickness; Restek Corp, Bellefonte, PA) was used for the separation of individual isomers. The column oven temperature was programmed from $100^{\circ} \mathrm{C}(1 \mathrm{~min})$ to $160^{\circ} \mathrm{C}(3 \mathrm{~min})$ at a rate of $4{ }^{\circ} \mathrm{C} / \mathrm{min}$, and then to $250^{\circ} \mathrm{C}$ at $3{ }^{\circ} \mathrm{C} / \mathrm{min}$, with a final hold time of $5 \mathrm{~min}$ for PCBs. For PBDEs, the column temperature was programmed from $100{ }^{\circ} \mathrm{C}(1 \mathrm{~min})$ to $160^{\circ} \mathrm{C}(3 \mathrm{~min})$ at a rate of $10^{\circ} \mathrm{C} / \mathrm{min}$, and then to $260^{\circ} \mathrm{C}$ at $2{ }^{\circ} \mathrm{C} / \mathrm{min}$, with a final hold time of $5 \mathrm{~min}$. The MS was operated in an electron impact $(70 \mathrm{eV})$, selected ion monitoring mode. PCB congeners were monitored at the two most intense ions of the molecular ion cluster. An equivalent mixture of Kanechlor (KC300, 400, 500, and 600) with known PCB composition was used in the identification of PCB congeners. Quantification of PCB congeners was based on external calibration standards containing known concentrations of di- through deca-CB congeners. Concentrations of individually resolved peaks of PCB isomers were summed to obtain total PCB concentrations. PBDE congeners were monitored at molecular ion clusters, $[\mathrm{M}]^{+}$and $[\mathrm{M}+2]^{+}$or $[\mathrm{M}+4]^{+}$. Eight major PBDE congeners 28, 30, 47, 85, 99, 100, 153, and 154 were targeted for analysis. Total PBDE concentrations represent the sum of all tri- through hexa-BDE congeners. PBDE congeners were quantified using an external calibration standard. PCB and PBDE congeners are represented by their IUPAC numbers.

Organochlorine pesticides were analyzed using a Agilent Technologies 6890N gas chromatograph-electron capture detector (GC-ECD). A capillary column coated with DB-5 (30 $\mathrm{m} \times 0.25 \mathrm{~mm}$ i.d. $\times 0.25 \mu \mathrm{m}$ film thickness) was used for the separation of pesticides. Concentrations were calculated from the peak area of the sample to that of the corresponding external standard. DDTs refers to the sum of $p, p^{\prime}$-DDE, $p, p^{\prime}$-DDT and $p, p^{\prime}$-DDD; chlordanes to 
the sum of cis-chlordane, cis-nonachlor, trans-nonachlor, and oxychlordane; HCHs to the sum of $\alpha-, \beta-$, and $\gamma$-isomers.

The extraction, clean-up, and fractionation steps were evaluated by measurement of the absolute recoveries of the compounds spiked and passed through the entire analytical procedure. Mean ( \pm standard deviation) recoveries of ${ }^{13} \mathrm{C}$-labeled PCB congeners \#30, 118, 153, and 194 spiked into the samples were $80 \pm 14 \%, 82 \pm 17 \%, 89 \pm 12 \%$, and $91 \pm 14 \%$, respectively. Overall recoveries of PCB congeners, including PCB 30, ranged from 85 to $115 \%$. Mean ( \pm standard deviation) recoveries of ${ }^{13} \mathrm{C}$-labeled PBDE congeners 28 and 47 were $92 \pm 14 \%$ and 91 $\pm 14 \%$, respectively. Overall recoveries of PBDEs ranged from 82 to $103 \%$. The reported concentrations were not corrected for recoveries. Recoveries of organochlorine pesticides through the analytical procedure ranged from 85 to $110 \%$. Procedural blanks were analyzed for every set of 10 samples, as a check for interferences. Calculated concentrations were reported as below the limit of detection, if either the observed isotope ratio was not within $\pm 20 \%$ of the theoretical-ratio, or the peak area was not greater than the specified threshold (3 times the noise). The detection limits of individual PCB and PBDE congeners varied from 40 to $400 \mathrm{pg} / \mathrm{g}$, wet wt, and for organochlorine pesticides they varied from 100 to $1000 \mathrm{pg} / \mathrm{g}$, wet wt.

Concentrations of perfluorinated acids in liver tissue were determined by the ion pairing liquid extraction method described elsewhere $(16,17) .{ }^{13} \mathrm{C}$ perfluorooctanoic acid $\left({ }^{13} \mathrm{C}-\mathrm{PFOA}\right)$ and perfluorobutanesulfonate (PFBS) were used as internal standards. One milliliter of $0.5 \mathrm{M}$ tetrabutylammonium hydrogen sulfate solution, $2 \mathrm{~mL}$ of sodium carbonate buffer $(0.25 \mathrm{M}, \mathrm{pH}$ 10), and $5 \mathrm{~mL}$ methyl-tert-butyl ether (MTBE) were added to the sample. After shaking for 30 min, the organic layer was separated by centrifugation, and the extraction was repeated with a further $5 \mathrm{~mL}$ of MTBE. The extracts were combined and evaporated to dryness under a gentle 
flow of nitrogen, before being reconstituted in $1 \mathrm{~mL}$ of methanol and vortexed. The extract was filtered through a $0.2-\mu \mathrm{m}$ nylon filter into an autosampler vial with polypropylene cap.

Separation of perfluorinated acids was performed using an Agilent 1100 high performance liquid chromatograph (HPLC). Ten microliters of the extracts were injected onto a $50 \times 2 \mathrm{~mm}(5 \mu \mathrm{m})$ Keystone Betasil $\mathrm{C}_{18}$ column. A gradient mobile phase of methanol and $2 \mathrm{mM}$ ammonium acetate was used. At a flow rate of $300 \mu \mathrm{L} / \mathrm{min}$, the mobile phase gradient was ramped from $10 \%$ to $25 \%$ methanol in $7 \mathrm{~min}$, then to $100 \%$ methanol at $10 \mathrm{~min}$, held at $100 \%$ methanol for $2 \mathrm{~min}$, and then ramped down to $10 \%$ methanol. For quantitative analysis the HPLC was interfaced with an Applied Biosystems API 2000 tandem mass spectrometer (MS/MS). The MS/MS was operated in electrospray negative ion mode. Analyte ions were monitored using multiple reaction monitoring (MRM) mode. Parent and daughter ion transitions monitored for PFOS, PFHS, ${ }^{13} \mathrm{C}-\mathrm{PFOA}$, PFOA, PFOSA, PFNA, PFDA, PFUA, and PFDoA were $499>99,399>80,370>170,413>169,498>78,369>219,513>219,563>169$, and 613>169, respectively. Quantitation was performed using a linear regression analysis weighted 1/x of a single unextracted calibration curve. Seven-point calibration curves were produced from 0.1 to $100 \mathrm{ng} / \mathrm{mL}$ concentrations. The coefficient of determination $\left(\mathrm{r}^{2}\right)$ for each calibration was > 0.99. Quality control standards were measured after every 10 samples to check for instrumental drift. Analysis was stopped, and a new calibration curve was run, if the quality control standard was not measured at $\pm 30 \%$ of its theoretical value. PFOA was consistently found in procedural blanks and methanol injections performed between samples. However, this background PFOA signal is consistent and can be subtracted from the calibration curves and samples. PFOA contamination can also be introduced from fluoropolymer containing vial caps (Yamashita et al., 2004). Polypropylene caps were used in this study.

All procedural blank peak areas were less than half the determined limit of quantitation (LOQ) for each analyte. The LOQ was estimated as three times the lowest concentration point on 
the calibration curve, which is accurately measured within $\pm 30 \%$ of its theoretical value. The LOQ was $1 \mathrm{ng} / \mathrm{g}$, wet wt, for all of the perfluorinated compounds. Recoveries of target compounds spiked into livers, and passed through the procedure, ranged between 60 and $145 \%$. Recoveries of ${ }^{13} \mathrm{C}$-PFOA ranged from 85 to $110 \%$. The reported concentrations were corrected for the recoveries of the internal standard.

\section{Supplementary Figures:}

Figure 5. Comparison of mean $( \pm \mathrm{SD})$ concentrations of organohalogens in livers of polar bears from the Beaufort Sea and Chukchi Sea subpopulations.

Figure 6. Composition (\%) of chlordane (CHL) compounds, HCH isomers, and DDT compounds in livers of polar bears from Alaska.

Figure 7. Comparison of PCB congener composition in livers of polar bears from the Beaufort and Chukchi Sea subpopulations. *Denotes significant difference $(\mathbf{p}<0.05)$.

Figure 8. Box and Whisker plot of PBDE 47 concentrations in livers of polar bears from the Beaufort Sea and Chukchi Sea subpopulations (circles represent mean, square represents outlier, and horizontal line represent median).

Figure 9. Comparison of mean $( \pm \mathrm{SD})$ concentrations of perfluorinated compounds in male and female polar bears from the Beaufort and Chukchi Sea subpopulations.

Figure 10. Box and Whisker plot of perfluorochemical concentrations in livers of polar bears from Beaufort Sea and Chukchi Sea subpopulations (circles represent mean, squares represent outliers, and horizontal line represent median; lower and upper bars for the "box" represent $25^{\text {th }}$ and $75^{\text {th }}$ percentiles and the error bars represent the $5^{\text {th }}$ and $95^{\text {th }}$ percentile).

Figure 11. Relationship between concentrations (ng/g, wet wt) of PFOS and PFNA, PFNA and PFDA, and PFDA and PFUA in livers of polar bears from Alaska. 


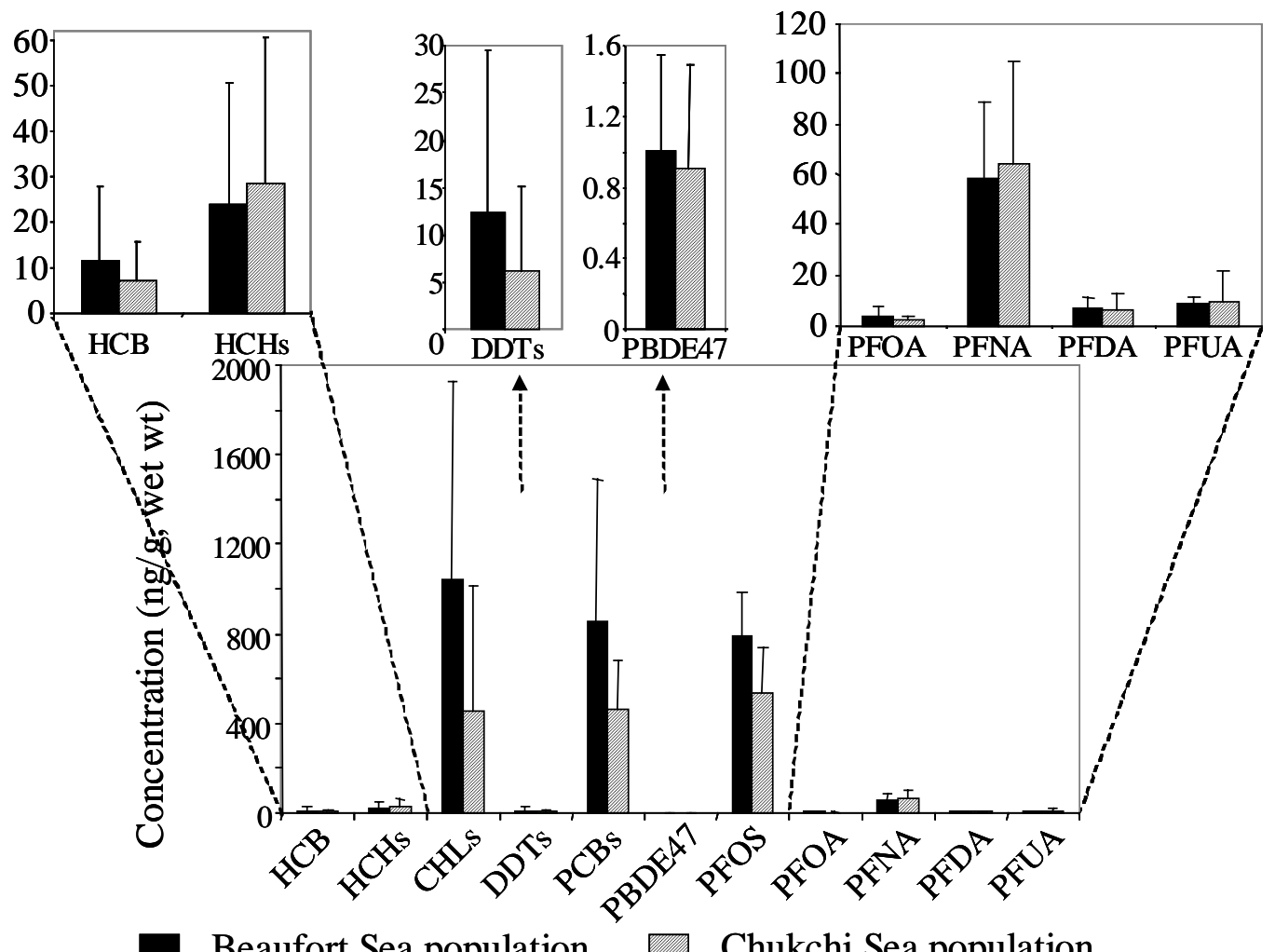

- Beaufort Sea population Chukchi Sea population 
Fig. 6, supplementary Kannan et al.

\section{CHLs}

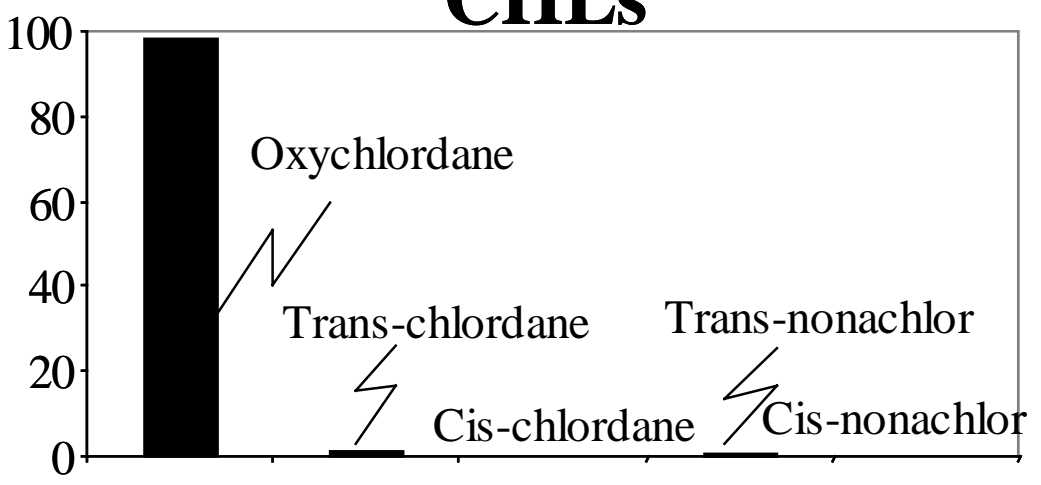

HCHs
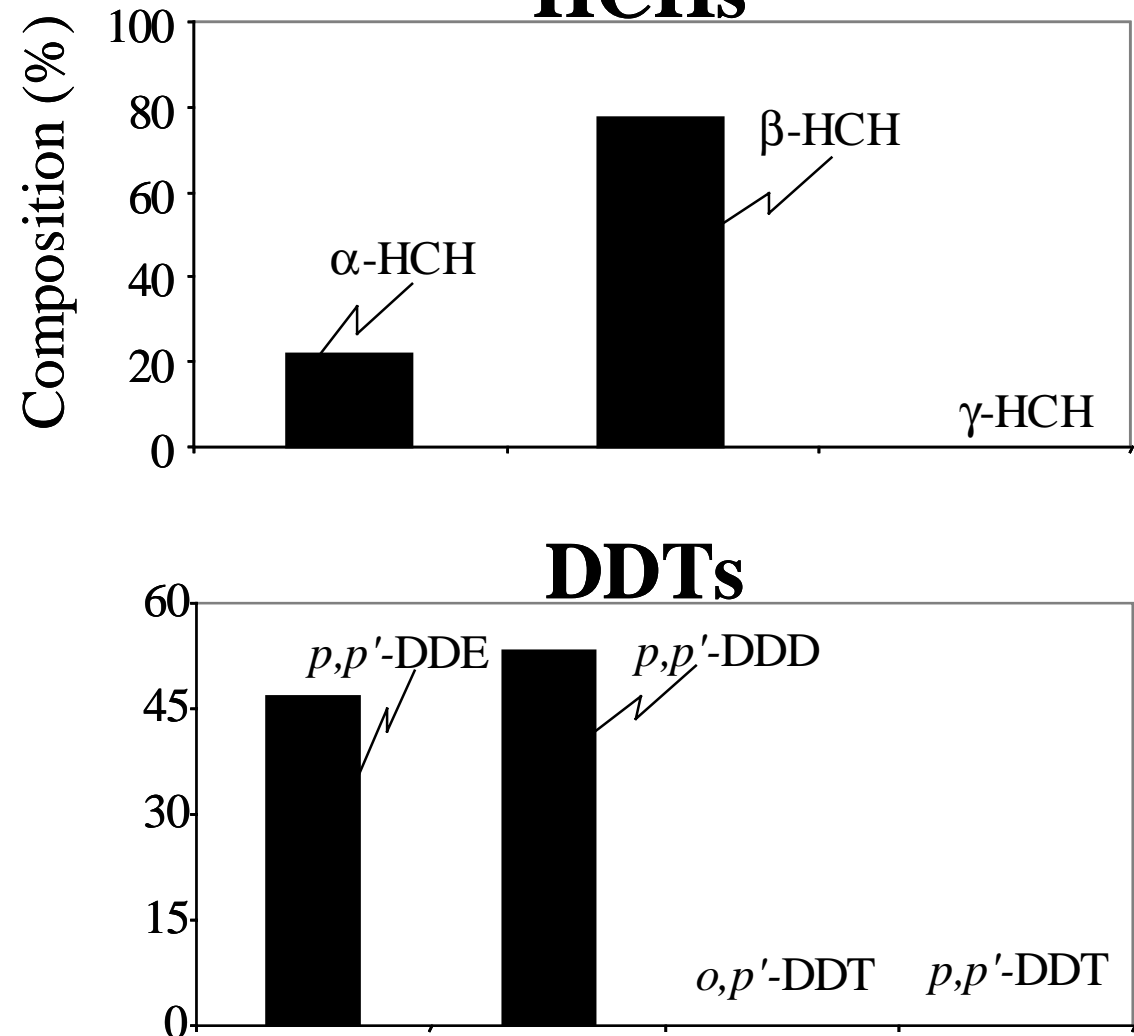


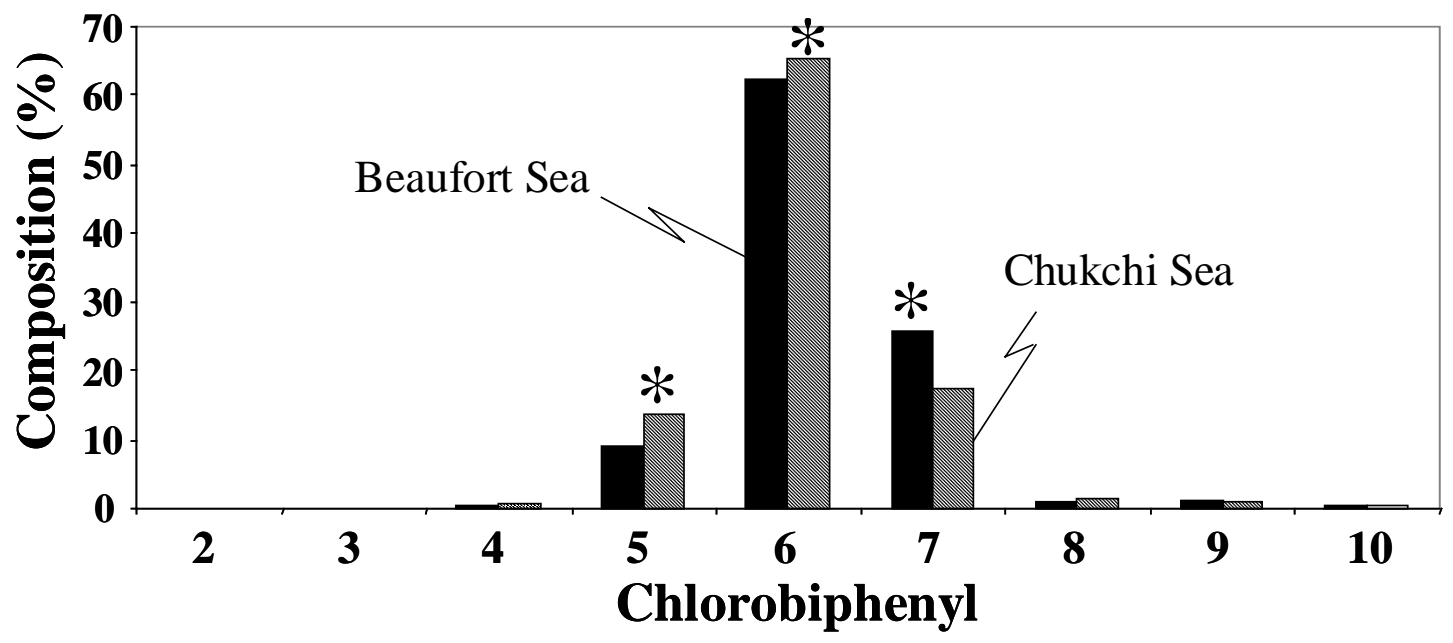

* Denotes significantly high $(\mathrm{p}<0.05)$ 
Fig. 8, supplementary

Kannan et al.

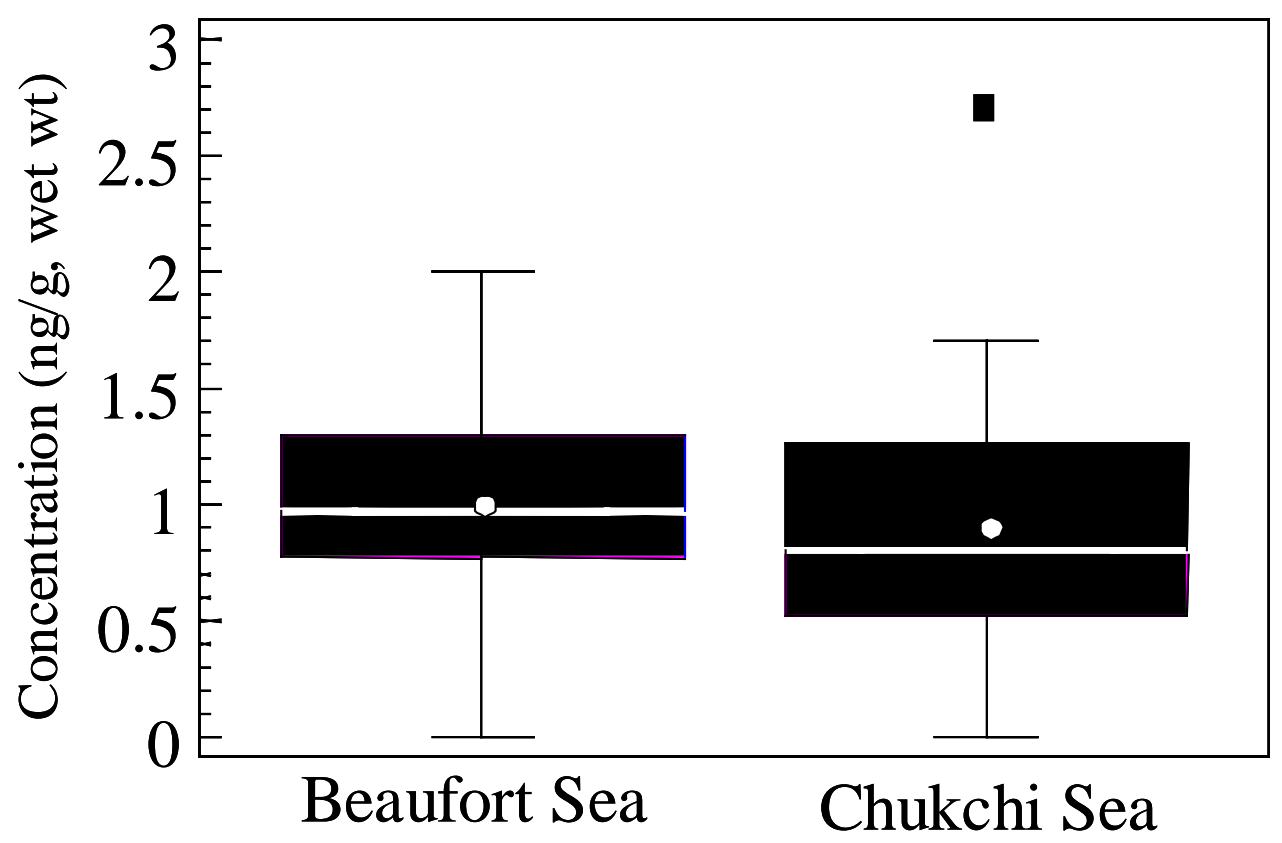


Fig. 9, supplementary

Kannan et al.

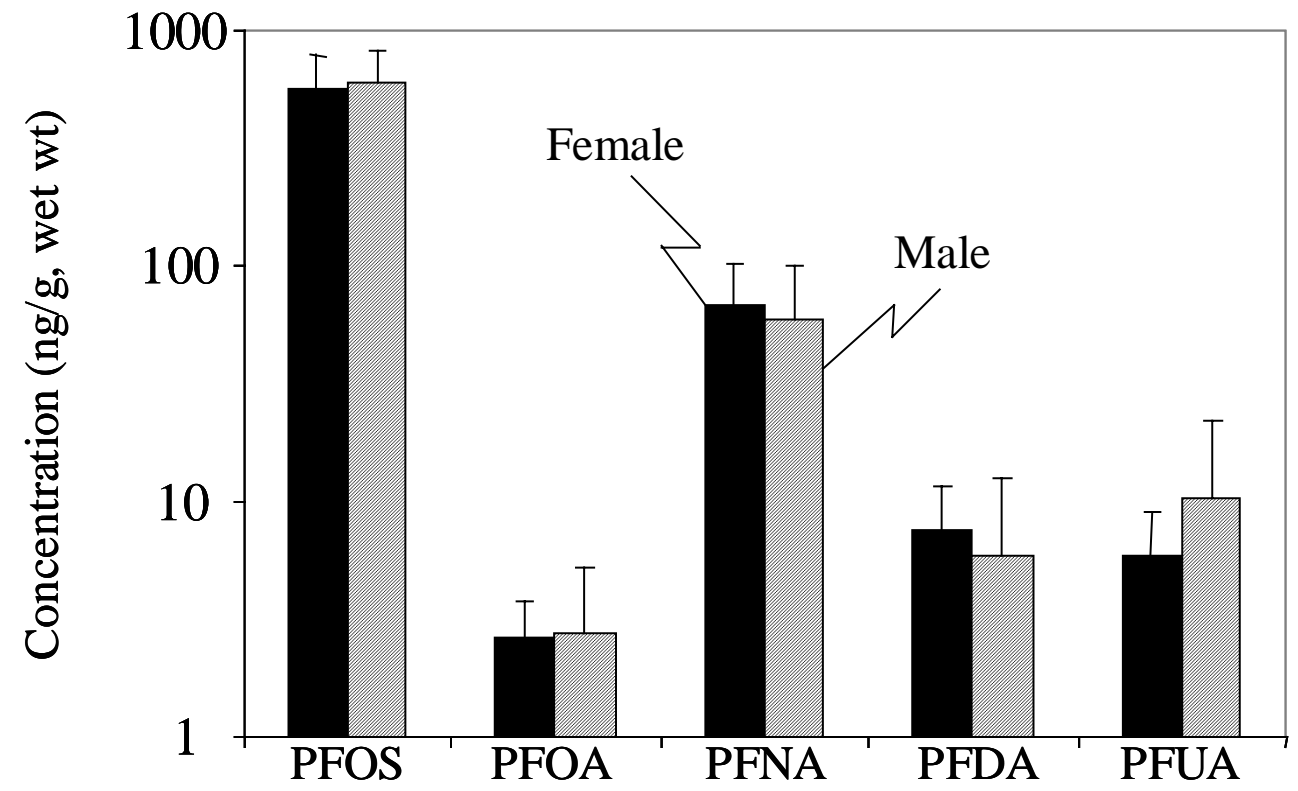


Fig. 10, supplementary

Kannan et al.
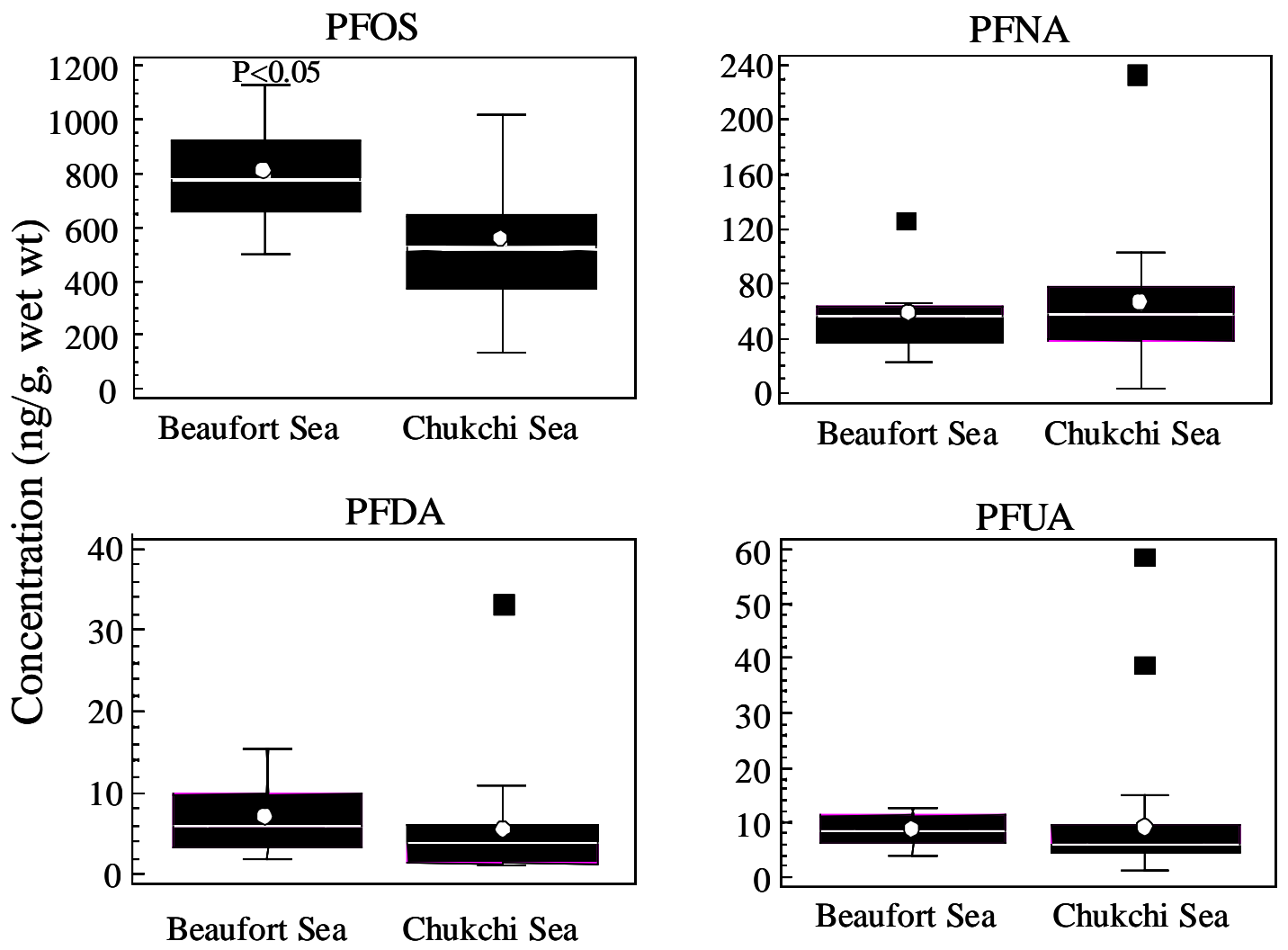

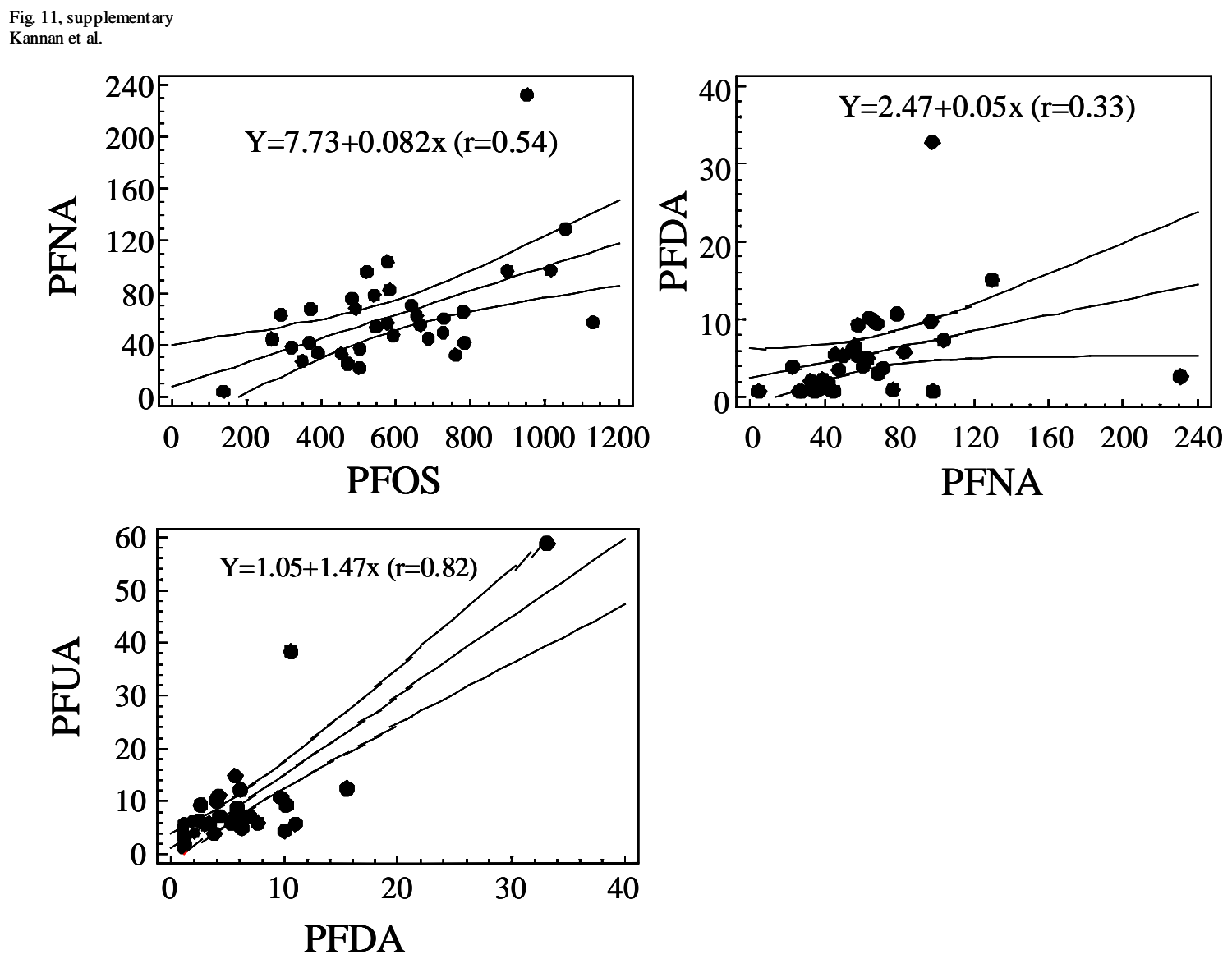